
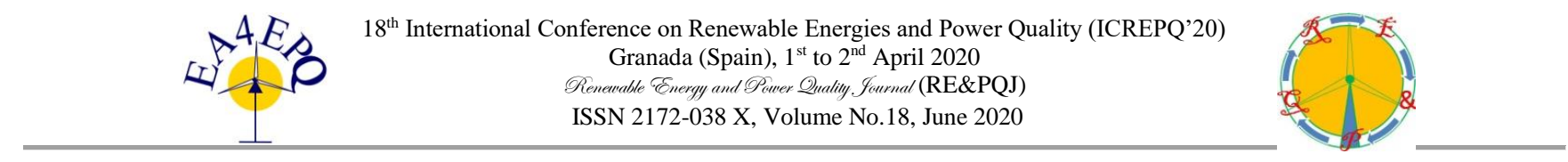

\title{
Simulation Technologies Applicable to Microgrids
}

\author{
R. B. Otto ${ }^{1}$, F. P. Silva ${ }^{1}$, M. B. do Carmo ${ }^{1}$, A. B. Piardi ${ }^{1}$ and R. A. Ramos ${ }^{2}$ \\ ${ }^{1}$ LASSE - Itaipu Technological Park \\ Foz do Iguaçu, Paraná (Brazil) \\ Phone/Fax number: +5545 35767089, e-mail: rodrigobueno@pti.org.br, lasse@pti.org.br \\ ${ }^{2}$ EESC - University of Sao Paulo \\ São Carlos, São Paulo (Brazil) \\ rodrigo.ramos@ieee.org
}

\begin{abstract}
The new paradigm of the electric sector, given the increased penetration of distributed generation, includes a growing number of microgrids. Therefore, the implementation of microgrids needs to be preceded by studies and simulations to evaluate the possible scenarios under which these systems will operate. This paper presents an overview of state-of-the-art technologies for microgrid simulation and describes an example implemented at the facility to which the first authors are affiliated.
\end{abstract}

Key words. Microgrid, Simulation Tools, Real-Time Simulation, Distributed Energy Resources, Hardware-inthe-Loop.

\section{Introduction}

The deregulation process of electrical power systems - that opened the way towards competitive markets with independent power producers - added to the necessities demanded by industrialized economies characterized by the diversification of energy matrix as well as the increasing of the efficiency in the process of generation and transport of electric power, provided the subsidies for the advent of distributed generation concept.

To take advantage of the potential benefits from in distributed generation, there is a necessity for a complete overhaul of electricity distribution systems through better use and complete automation of their elements, giving rise to the concept of microgrids [2] [3]. For the fact that these systems can be considered a basic feature of future distribution systems, and due to its inherent operation complexity, it becomes mandatory to characterize their behavior and performance, both in the design phase as well as during their operation.

In the context previously presented, in this paper, a brief contextualization of microgrids will be presented, followed by a review of the technologies applicable to the simulation and testing of microgrids. To validate the proposal a microgrid model commonly referenced in the technical literature was modeled on a real-time simulation platform.

\section{Microgrids}

Microgrids are defined as distributed electrical systems containing loads and distributed energy resources - DER (such as distributed generators, energy storage systems and controllable loads) that can be operated in a controlled and coordinated manner, connected to or isolated from the main network [4].

The main reason for using a microgrid is to incorporate the full potential of distributed generation sources into the electrical system [1] [6], using these resources intelligently and efficiently.

Figure 1 presents the general architecture of a microgrid, which can be divided into three main layers, reflecting the challenges for microgrids implementation [2] [3] [4]:

1. First layer - constituted by the power equipment (electrical system). The main challenges of this layer are: Integration and operation of DER;

2. Second layer - constituted by the communication infrastructure. The main challenges of this layer are: Integration of equipment with different protocols, availability, reliability, scalability and cybersecurity;

3. Third layer: constituted by the high-level control system, which contains the general controllers and Human-Machine Interfaces (HMIs). The main challenges of this layer are: Optimization and Coordination of DER.

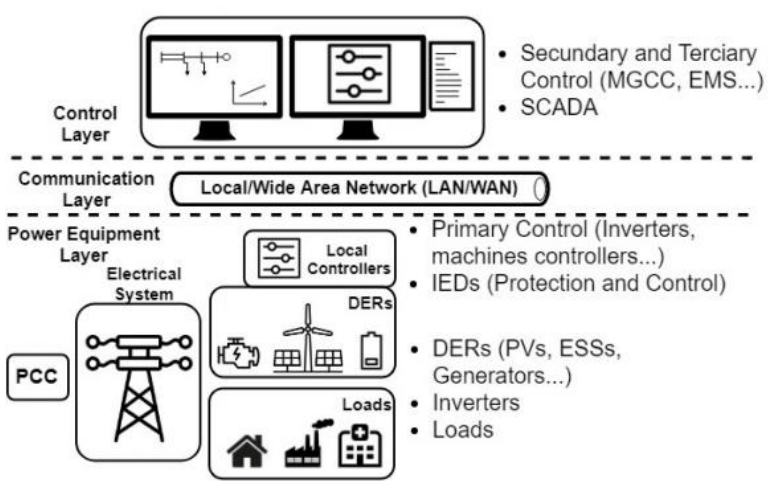

Figure 1 - The general architecture of a microgrid [5] 
According to [7], we have advantages in the use of microgrids from the economic, technical and environmental point of view.

I. Economic: the possibilities of profit due to power generation by the owners of distributed generation sources, the creation of new business models for the distribution concessionaires, and the increased efficiency in the processes of generation and consumption of electrical energy.

II. Environmental: reduction of greenhouse gases by promoting the use of renewable energy sources.

III. Technical: relief of the main system overload, support in the frequency and voltage regulation, reduction of losses and the increase of reliability of the power supply to end consumers.

Because microgrids are considered a reality for future power systems, in addition to their inherent operation complexity, it is mandatory to characterize their behaviors and performances, both in design and operation phases [7]. In this context, computer simulations favor the analysis and verification of many concepts and schemes related to microgrids [8].

\section{Simulation Tools}

There are several tools for simulation and analysis of microgrid. The use of computer simulators, tools that allow the study and testing of various microgrids architectures and operational scenarios, make it easier to understand the phenomena and concepts surrounding these systems [9].

GridLAB-D is an open-source software. It was developed by the US Department of Energy (DOE) through Pacific Northwest National Laboratory (PNNL). It is purely command-line software (without Graphical User Interface (GUI)), where the simulation is flexible and based on agent modeling. The input files (.GLM and .XLM) are processed and generate the simulation result in output files. This allows large systems to be efficiently simulated. This software can also be seen as a collection of modeled objects that represent the various elements present in distribution systems [11].

The company responsible for developing the Hybrid Optimization Model for Multiple Energy Resources (HOMER) was founded in 2009 by the National Renewable Energy Laboratory (NREL). HOMER Energy sells two products, HOMER PRO and HOMER GRID. Both products do the technical optimization and find the lowest cost system that meets technical restrictions imposed by the application [12].

MATLAB is a proprietary software, which has MATLAB Simulink as its simulation platform. Among the simulation tools, MATLAB Simscape Electrical is the solution for the simulation of electronic components, mechatronics, and power systems. Usually, this tool is referred by the name of SimPowerSystems, and many publications still referring to this name [13].
Open Distribution System Simulator (OpenDSS) is an open-source software for the simulation of power distribution systems. Since 1997 it is maintained by Electric Power Research Institute (EPRI), and its purpose is to facilitate studies of DER interconnection in the context of smart grids [14].

Power Systems Computer Aided Design (PSCAD) is a tool for simulation of electromagnetic transients. Developed in 1976, the program is a user-friendly interface, widely recognized to perform electromagnetic transient analyses. The visual environment provided by PSCAD supports the interfaces of circuit modeling, runtime control, analyses and report generation [17].

The increase and importance of microgrids have brought a demand for study tools and sophisticated Hardware-inthe-Loop (HIL) testing facilities to evaluate the operation of associated control and power devices in the grid [10]. The great advantage of the Real-Time Simulator (RTS) platforms is to allow the possibility to interface external devices - usually referenced as Devices Under Test (DUTs) [5], [15] ,[16]. Three real-time simulation platforms were analyzed: OPAL-RT, Typhoon HIL, and RTDS.

Founded in 1997, OPAL-RT Technologies is a Canadian company that markets RTS to meet the demands of industry and research institutes. The OPAL-RT simulation platform uses two main software: HYPERSIM and RT-LAB [18]. To better understand the differences between simulation platforms, OPAL-RT provides a comparative table in [20] and a catalog in [19]. In [20], related information is provided in the above paragraphs in a more organized manner, as well as specific applications with each platform. In [19], a comparative graph is presented between the four solutions based on the frequency of the phenomena represented and the number of nodes of the simulated system.

Founded in 2008, North American Typhoon HIL Inc. sells three series of RTS platforms: 4, 6 and M. The series 4, model HIL402, has the size of a notebook and uses a software called HIL Control Center. Series 6 is more focused on power electronics. With up to six cores, the HIL602 weighs approximately $4 \mathrm{~kg}$. On the other hand, Series $M$ is specifically intended for testing controllers and has several sizes. The software includes the following modules: Schematic Editor, for modeling the circuits; HIL SCADA, which allows the user to reconfigure the testbed; Test Suite, which comprises a certification program; and Power Systems Toolbox, which comprises a library specially developed for microgrid analyses [21].

Real-Time Digital Simulator (RTDS) refers to the RTS platform produced by Canadian company RTDS Technologies. The company was responsible for introducing the first fully digital power system simulator in 1989. As a result, RTDS has become a world reference for the simulation of power systems in real-time. The main applications are in the areas of: microgrid/smart grid simulation, cyber-security, Power Hardware-in-the 
Loop (PHIL), High Voltage Direct Current (HVDC) systems, Flexible Alternate Current Transmission Systems (FACTS), protection systems testing, power electronics, control systems testing, Phasor Measurement Units (PMUs) testing, IEC 61850, and DER integration. Figure 2 illustrates the application of RTDS simulator in microgrids analyses, where is enabled the testing of physical equipment in the same conditions encountered in real applications [10]. To construct the models a proprietary software package denominated Real-time Simulation Computer Aided Design (RSCAD) is used. This software package was developed to provide a fully graphical interface to the RTDS, making easier the operation of the real-time simulation platform.

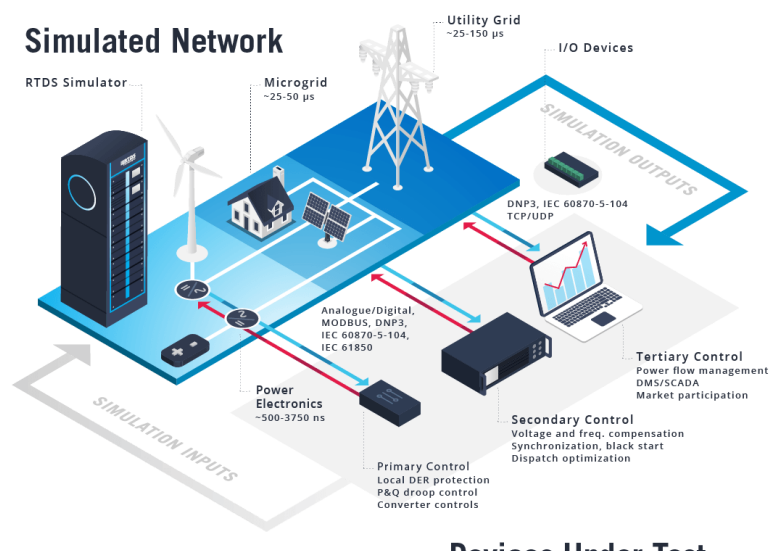

Devices Under Test

Figure 2 - HIL testing of microgrid control systems using RTDS [10]

Due to the potentialities pointed out, added to the equipment availability at the Itaipu Technological Park laboratorial infrastructure, the simulation tool used in this article was the RTDS platform.

\section{Example of Microgrid Implementation in a RTS Platform}

This section presents an actual implementation of a benchmark microgrid model in a real-time simulator performed at the facility to which the first authors of the paper are affiliated. This implementation represents entirely the microgrid through digital models in the simulation environment, being feasible to exchange data with peripheral equipment (such as controllers) via communication protocols or analog interface, closing the loop in the simulation [2].

The model implemented in this work is a modified version of the CIGRE Benchmark Microgrid - Medium Voltage which single line diagram is shown in Figure 3. This modified version was originally presented in [22].

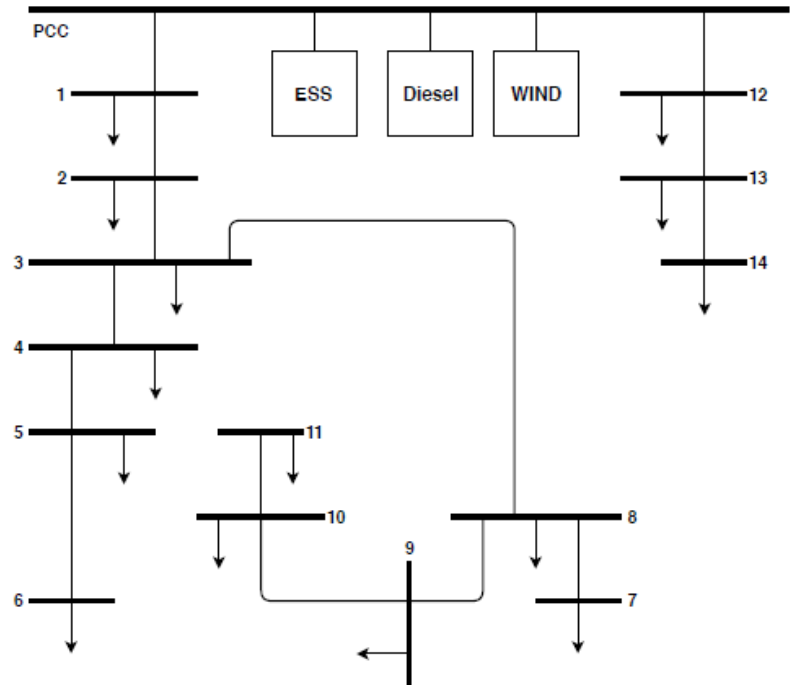

Figure 3 - Single line diagram of the CIGRE Benchmark Microgrid - Modified Version

In general guidelines, the microgrid of Figure 3 operates permanently isolated from the bulk power system and has three sources: Energy System Storage (ESS), diesel generator and wind generator, both connected to point of common coupling (PCC). The files of this implementation in RTDS format is available in [23].

\section{A. Diesel Generator}

The diesel generator was modeled as a salient pole synchronous machine. For this purpose, a model from the RSCAD library was used.

The IEEE AC1 excitation model present in the software library RSCAD was used and adjusted according to [24].

The speed regulator was modeled with isochronous characteristic since it performs the frequency control of the microgrid. The block diagram of the implemented control system is presented in Figure 4.

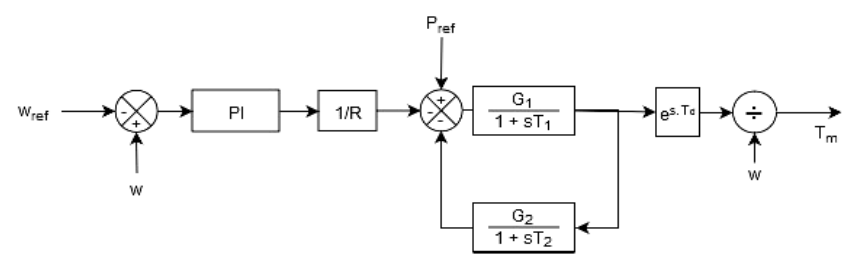

Figure 4 - Block diagram of speed regulator of the diesel generator

\section{B. Wind Generator}

The model of the wind generator used for the simulations was the Doubly-Fed Induction Generator (DFIG). The DFIG consists of an induction machine round rotor, with the stator connected to the grid via a transformer unit, and the rotor powered via a back-to-back converter, as shown in Figure 5 [25], [26]. 


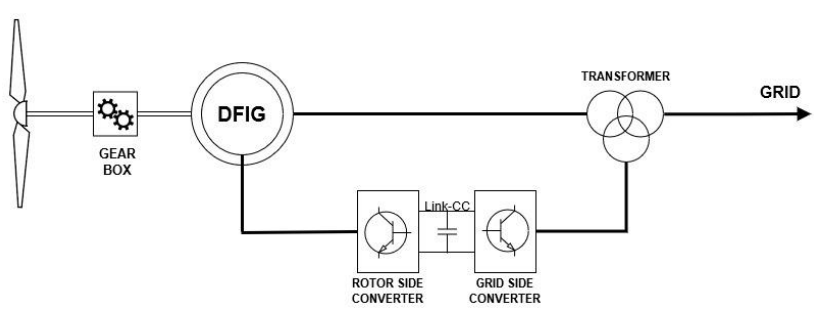

Figure 5 - Doubly-fed induction generator

The DFIG model has gained importance due to the technological advances in the power electronics area, and the development of vector control techniques. This arrangement allows the decoupling between active and reactive power, as well as the generation of power at rated frequency and voltage regardless of the rotor speed [25], [26].

The back-to-back converter controls the power flow between the rotor and the grid. This converter consists of two inverters with Insulated Gate Bipolar Transistors (IGBTs), sharing a direct current link as illustrated in Figure 6 [25], [26].

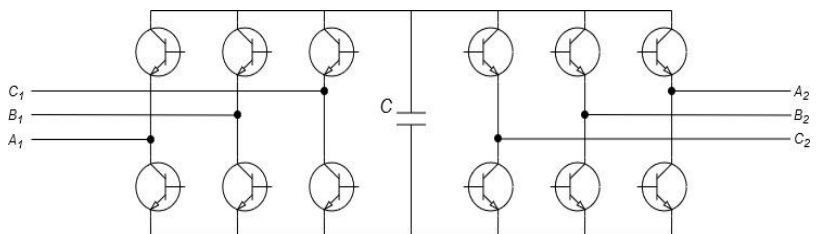

Figure 6 - Back-to-back converter circuit

In this work, the detailed back-to-back converter model was implemented. In other words, the IGBTs keys were properly represented as illustrated in Figure 6.

For proper operation of this converter two control systems are required, namely the Rotor Side converter Controller (RSC), and the Grid Side converter Controller (GSC). RSC is responsible for control the active and reactive powers provided by the wind generator, while GSC is responsible for maintaining constant DC-link voltage, to provide reactive power support, and to control the power exchange between the grid and the converter. More information about these controllers can be found in [25], [26].

\section{Energy Storage System}

The battery model used was the Li-Ion Battery technology - which is present in the RSCAD library - connected to the network via a Voltage Source Converter (VSC).

The VSC was implemented using an averaged model with dependent sources, as illustrated in Figure 7. As it neglects the effects of thyristor switching [27], the averaged model was used due to the processing limitations of the real-time simulation structure available for testing.

According to [27], although it is not a true representation of the DC/AC converter, the model used does not show significant losses when compared to the detailed model if the microgrid operates under load and with balanced conditions.

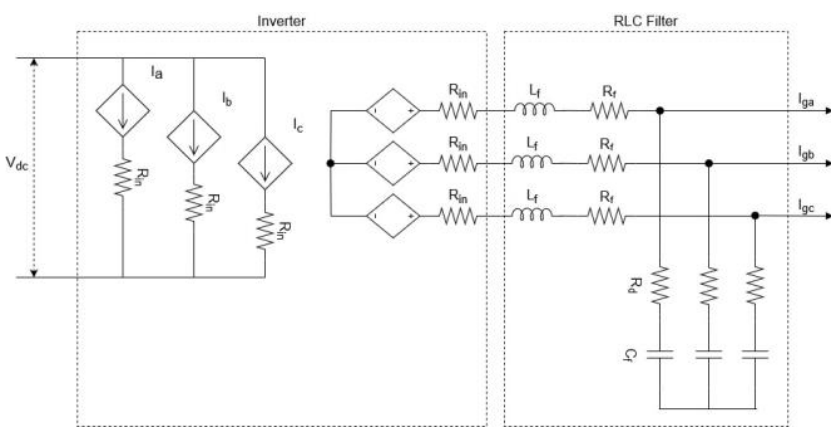

Figure 7 - Back-to-back converter circuit

The used battery control is based on the $\mathrm{d}-\mathrm{q}$ axis decoupling for the control of the active and reactive power supplied to the grid. Figure 8 illustrates the block diagram of the battery control.

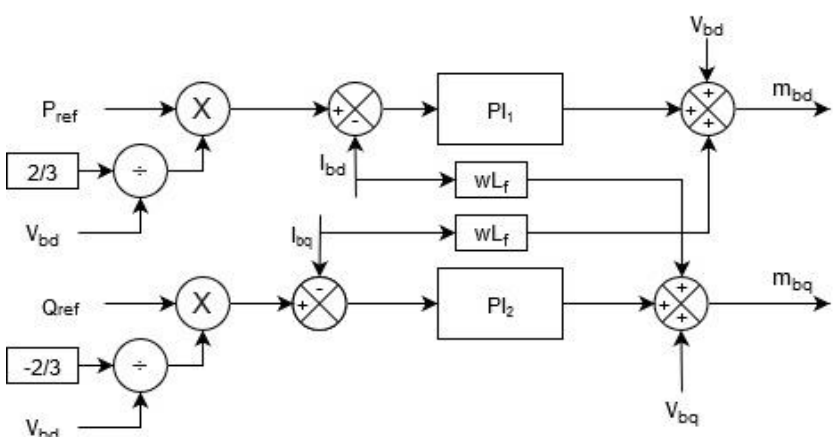

Figure 8 - Block diagram of the battery control

\section{Transmission Lines}

Due to their small lengths, the transmission lines were modeled using their PI equivalents. The electrical parameters of the lines can be found in [22].

\section{E. Loads}

All loads of the microgrid are three-phase, balanced and were modeled as dynamic loads. This model is available in RSCAD software package. The electrical parameters of the lines can be found in [22].

\section{Tests and Results}

In this section, the results obtained from the initial tests performed in the implemented microgrid are shown. Due to processing limitations, the global simulation time-step was set to $90 \mu \mathrm{s}$. Furthermore, it was adjusted a sub-time step of $3.6 \mu \mathrm{s}$ to simulate the power electronics converters.

In order to analyze the dynamic performance of the system, three simulations of events held to test the implemented microgrid, which will be described below. In both simulations, the diesel generator supplied approximately $0.2 \mathrm{MW}$ of active power and $0.3 \mathrm{Mvar}$ of reactive power, the battery bank provided approximately $0.2 \mathrm{MW}$ of active power, and the wind generator approximately $0.7 \mathrm{MW}$ of active power. 
Each simulation performed differs in the level of the load step applied at Bus 9. The load step applied in each simulation is presented in Table I.

Table I - Load steps for the simulated events

\begin{tabular}{c|c|c}
\hline Simulation & $\begin{array}{c}\text { Active Power } \\
{[\mathrm{MW}]}\end{array}$ & $\begin{array}{c}\text { Reactive Power } \\
{[\mathrm{MVar}]}\end{array}$ \\
\hline 1 & 0.34 & 0.036 \\
\hline 2 & 0.5 & 0.051 \\
\hline 3 & 0.5 & 0.51 \\
\hline
\end{tabular}

Also, it was considered that there is an extremely important load connected to bus 11 , so this bus was considered the main bar of the system.

Figure 9 shows the results of frequency and voltage at bus 11 for the simulated events. It is verified that the microgrid is stable from the viewpoint of simulated events, and the electrical quantities reach adequate values after the perturbations. It is important to highlight that the results presented in Figure 9 can be externalized to test the equipment present in the control center (third layer) to define and validate the best control strategy.
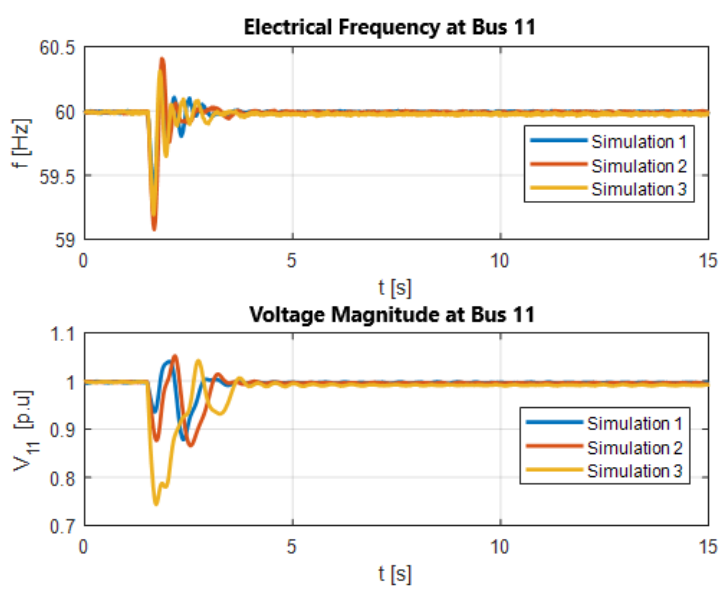

Figure 9 - System frequency and voltage at bus 11 for the simulated events

\section{Conclusion}

Because it is a multidisciplinary and highly complex theme, the complete understanding of the phenomena that permeate the concept of microgrids demands the application of sophisticated analysis tools. Within this context, this paper presented a comprehensive survey of the main tools used for this task.

Among the presented tools, emphasis is given to those involving the concept of HIL simulation. Through the use of real-time simulation platforms and by establishing appropriate interfaces between simulated and real parts of the system, the HIL concept allows equipment to be tested in conditions very close to those found field, with the advantage of analysis flexibility through the possibility of representation of different operational conditions.

The results from the presented example, which correspond to an actual implementation at the facility to which the first authors are affiliated, illustrated the previously mentioned advantages of having a flexible testbed for microgrid simulation.

\section{Acknowledgement}

We are grateful for the support of Itaipu Binacional and Itaipu Technological Park. This work is part of the research group "Microgrids and Systems with Distributed Generation by Real Time Simulation" (CNPQ) and "Hybrid Intelligent Electrical Microregions with High Penetration of Renewable Energies" (CYTED717RT0533).

\section{References}

[1] R. H. Lasseter, "Microgrids". IEEE Power Engineering Society Winter Meeting. (2002.) v. 1, p. 305-308 vol.1..

[2] A. B. Piardi, R. B. Otto , D. G. Sonoda, F. C. Santos, L.R.A Ferreira and R. A. Ramos, "Laboratory for Analysis of Microgrid with Real Time Simulation", ICREPQ'19 (2019).

[3] Working Group C6.22 "Microgrids Evolution Roadmap, Microgrids 1 Engineering, Economics, \& Experience" CIGRE (2015).

[4] A. Bani-Ahmed and A. Nasiri, "Development of Real-Time Hardware-in-the-Loop Platform for Complex Microgrids," ICRERA (2015), Palermo, pp. 994-998.

[5] A. B. Piardi, F. C. dos Santos, D. G. Sonoda, R. B. Otto, "Aspects of a Hybrid and Flexible Microgrid Laboratory Implementation”, XIV SEPOPE (2018).

[6] Marnay, C. et al. "Microgrid evolution roadmap". EDST (2015). p. 139-144.

[7] N. Hatziargyriou, et al. "Microgrids". IEEE Power and Energy Magazine (2007). v. 5, n. 4, p. 78-94.

[8] B. Xiao, et al. "Development of hardware-in-the-loop microgrid testbed". IEEE Energy Conversion Congress and Exposition (2015).

[9] P. P. Domingues, et Al. "Uso de Software Livre em Atividades de Ensino e Pesquisa em Microeletrônica". XLIV COBENGE (2016).

[10] RTDS Technologies, "Microgrid Simulation and Testing". Available in: < https://www.rtds.com/ >. Access in 2019.11.11.

[11] D. P. Chassin, K. Scheneider, C. Gerkensmeyer. "Gridlab-D: An opensource power systems modeling and simulation environment". T\&D (2008). p. 1-5. ISSN 2160-8555.

[12] HOMER. HOMER PRO and HOMER GRID. 2019. Available in: < https://www.homerenergy.com >. Access in 2019.11.11

[13] MATLAB Simscape Electrical. 2019. Available in: $<$

https://www.mathworks.com/products/simscapeelectrical .html >. Access in 2019.11.11

[14] EPRI. "Introduction to the OpenDSS". (2009). Available in: <http:// https://www.epri.com/\#/pages/sa/opendss?lang=en-US/>. Access in: 2019.11.11

[15] W. Ren, M. Steurer and T. L. Baldwin, "Improve the Stability and the Accuracy of Power Hardware-in-theLoop Simulation by Selecting Appropriate Interface 
Algorithms," in IEEE Transactions on Industry Applications, vol. 44, no. 4, pp. 1286-1294, July-Aug. 2008.

[16] J. Wang, Y. Song, W. Li, J. Guo and A. Monti, "Development of a Universal Platform for Hardware Inthe-Loop Testing of Microgrids," in IEEE Transactions on Industrial Informatics, vol. 10, no. 4, pp. 2154-2165, Nov. 2014.

[17] PSCAD. "User's Guide on the use of PSCAD" (2018). Available in: <https://hvdc.ca/uploads/knowledge base/pscad manual v4 6.pdf?t=1528395602>. Access in: 2019.11.13

[18] OPAL-RT. "Simulator Platform Comparison Chart" (2019). Available in: <https://www.opalrt.com/wpcontent/themes/ enfoldopal/pdf/L00161 0472.pdf>. Access in: 2019.11.13

[19] OPAL-BROCHURE. "Real-Time Simulation Solutions for Power Grids and Power Electronics" (2019). Available in: <https://www.opalrt.com/wpcontent/themes/enfoldopal/pdf/

L001610260.pdf>. Access in: 2019.11.13

[20] OPAL-CHART. "Website da OPAL-RT" (2019). Available in: <https://www.opal-rt.com/>. Access in: 2019.11.13

[21] TYPHOON. Website da Typhoon HIL. 2019. Available in: <https://www.typhoon-hil.com/>. Access in: 2019.11.13

[22] CIGRE Task Force C6.04.02, Benchmark Systems for Network Integration of Renewable and Distributed Energy Resources. CIGRE, (2014).

[23] R. B. Otto, R. F. Espinoza, A. Piardi, and M. do Carmo. "Microgrid benchmark system: Rtds implementation,". Available in: <https://github.com/Itaipr/MICROGRID-RTDS> (2019) [24] "IEEE recommended practice for excitation system models for power system stability studies," IEEE Std 421.5-2016 (Revision of Std 421.5-2005), pp. 1-207, Aug 2016.

[25] G. Abad, J. Lopez, M. Rodriguez, L. Marroyo, and G. Iwanski, Doubly fed induction machine: modeling and control for wind energy generation.

[26] H. Abu-Rub, M. Malinowski, and K. Al-Haddad, Power electronics for renewable energy systems, transportation and industrial applications.

[27] M. Farrokhabadi, S. Konig, C. A. Canizares, K. Bhattacharya, and T. Leibfried, "Battery energy storage system models for microgrid stability analysis and dynamic simulation," IEEE Transactions on Power Systems, vol. 33, no. 2, pp. 2301-2312, March 2018. 